\title{
ATLAS Event Store and $1 / 0$ developments in support for Production and Analysis in Run 3
}

\author{
Marcin Nowak $^{1, *}, \dagger$, Peter van Gemmeren ${ }^{2}$, and Jack Cranshaw ${ }^{2}$ \\ ${ }^{1}$ Brookhaven National Laboratory, Upton, New York 11973, USA \\ ${ }^{2}$ Argonne National Laboratory, Argonne, Illinois 60439, USA
}

\begin{abstract}
During the long LHC shutdown, ATLAS experiment is preparing several fundamental changes to its offline event processing framework and analysis model. These include moving to multi-threaded reconstruction and simulation and reducing data duplication during derivation analysis by producing a combined mini-xAOD stream. These changes will allow ATLAS to take advantage of the higher luminosity at Run 3 without overstraining processing and storage capabilities. They also require significant improvements to the underlying event store and the $\mathrm{I} / \mathrm{O}$ framework to support them. These improvements include: 1) an overhaul of the Run $2 \mathrm{I} / \mathrm{O}$ framework to be thread-safe and minimize serial bottlenecks, 2) introduction of new immutable references for object navigation, which don't rely on storage container entry number so data can be merged in-memory, 3) using filter decisions to annotate combined output stream to allow for fast event selection on input and 4) selecting optimized compression algorithms and settings to allow efficient reading of event selections.
\end{abstract}

\section{Introduction}

The ATLAS[1] experiment is one of the 4 experiments collecting data from the LHC at CERN. The LHC operation schedule consists of periods of activity (Runs) interspaced with breaks intended for hardware maintenance and upgrades (Long Shutdowns). Each consecutive Run generates an increasing amount of ever more complex physics data that needs to be processed. In order to keep up with the increasing processing needs, the experiments modify their software frameworks to utilize the available CPU resources in the most efficient way. The current systems, with large amounts for CPU cores but limited percore memory, compel migration to multi-threaded applications. In consequence, the I/O components have to evolve as well - to handle the resulting higher data rates and to function effectively in the multi-threaded environment.

\footnotetext{
* Corresponding author: mnowak@bnl.gov

$\dagger$ Copyright 2020 CERN for the benefit of the ATLAS Collaboration. Reproduction of this article or parts of it is allowed as specified in the CC-BY-4.0 license.
} 
The upcoming LHC Run 3 and especially Run 4 will require significant software performance improvements. In this paper we describe the developments in the ATLAS Event Store and the I/O framework that were done to meet the new requirements.

\section{Major software improvements}

Taking advantage of the currently on-going Long Shutdown 2, the software team is implementing 4 major improvements to the ATLAS offline event processing framework Athena[2] and analysis mode.

\subsection{Thread safety and concurrency in the $\mathrm{I} / \mathrm{O}$ framework}

One of the biggest changes in the Athena framework for Run 3 is the introduction of parallel Event processing using multi-threading (AthenaMT)[3]. In AthenaMT multiple Events are processed at the same time, each in a dedicated Event Store, and then written out (Figure 1). The I/O framework had to be adapted to accept overlapping write requests coming from the Stores, providing thread safety and allowing as much concurrency as possible in order to ensure good performance. Thread safety was achieved by identifying and protecting, with thread locking mechanisms, the critical sections of the components that have only once instance in the application. Examples are the central Conversion Service and accompanying POOL Service, which are responsible, respectively, for data object schema conversion between their transient and persistent representations and for organizing objects inside ROOT files in the POOL/APR file format[5]. It was also necessary to eliminate state information stored in these components. Concurrent execution was achieved by creating multiple copies of components that can operate independently and by allowing subcomponents, already existing in multiple instances (e.g. converters specialized by converted object type), to work in parallel.

Certain natural restrictions to concurrency come from the employed storage technology - in Athena writing to a given output file is done one Event at a time, to preserve integrity. Concurrent writing can still be performed on different files.

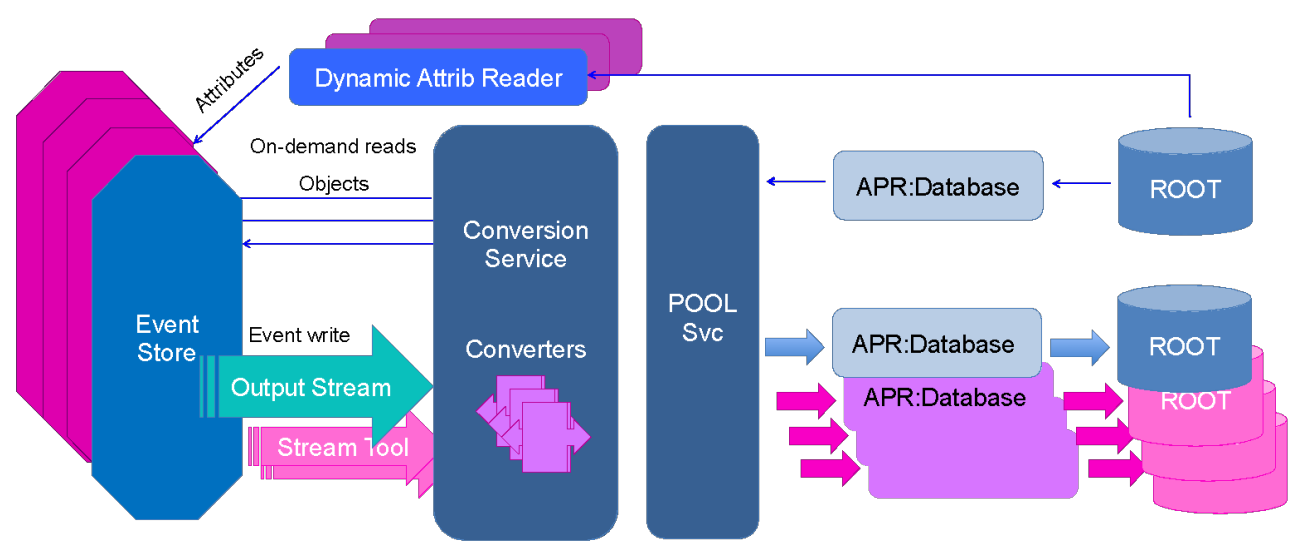

Figure 1. I/O Framework evolution from Single-Process (blue) to Multi-Threaded (all).

Reading Events from input is done sequentially in AthenaMT. However, certain data elements are read on-demand during processing and such requests can happen concurrently. If the requests demand access to the same file, they are serialized. 


\subsection{Object indexing in the storage layer}

Athena persistent storage technology (POOL/APR) is based on the concept that every data object (in the $\mathrm{C}++$ sense), when it is stored, it is assigned an immutable reference that can be used to retrieve it later. Internally, these references are broken into 2 parts: the object ID in its storage container and all information about that container, which is the same for all objects in a given container. Because of the repetitiveness and the size of container descriptions, they are stored in a helper "Links" table as a means to reduce space usage. Object references can then be represented just as a pair of two numbers - an object ID and an index into the Links table - called a POOL/APR Token [Figure 2].

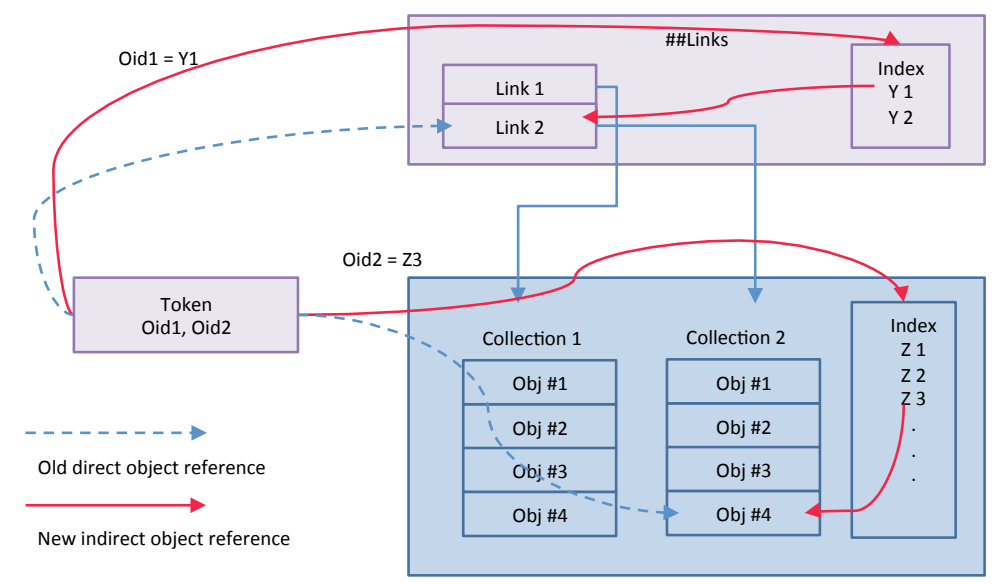

Figure 2. Indirect object access with indexing. An object reference (Token) is represented as a pair of identifiers (oid1, oid2). Oid1 points to an entry in the Links table describing containers, Oid2 enumerates an object in that container.

There are two production use cases where having direct object references is a disadvantage - file merging and concurrent writing to the same file. When merging files, the objects change their position and thus their object ID. During concurrent writing, the final object position is not known (due to buffering and delayed writing). To address both issues, we added an additional level of indirection for the Tokens in the form of in-file index (TTreeIndex). The index can be automatically updated by ROOT[4] during file merging, while the original Tokens stay unchanged. For concurrent writing, having an indirection layer allows to pre-assign logical object IDs that are later redirected to the real object IDs.

\subsection{Combined output stream with Event tagging}

The ATLAS Run 3 production model assumes using a single combined derivation output stream in order to avoid event data duplication, observed among Run 2 separate streams, and save disk space. Events in the common stream are marked as belonging to any of the derivation streams. For every Event additional values relevant to stream assignment decision can be stored. The most essential Event identification information in the form of a mini-EventInfo is also present (Figure 3). 
Keeping this information outside the main Event body in a simple, easily readable ROOT format, permits the Main Event Loop to efficiently read only events belonging to the desired derivation stream, with additional possibilities for selection using the stored attributes. The selectivity of typical ATLAS derivation jobs varies between $5 \%$ and $0.1 \%$.

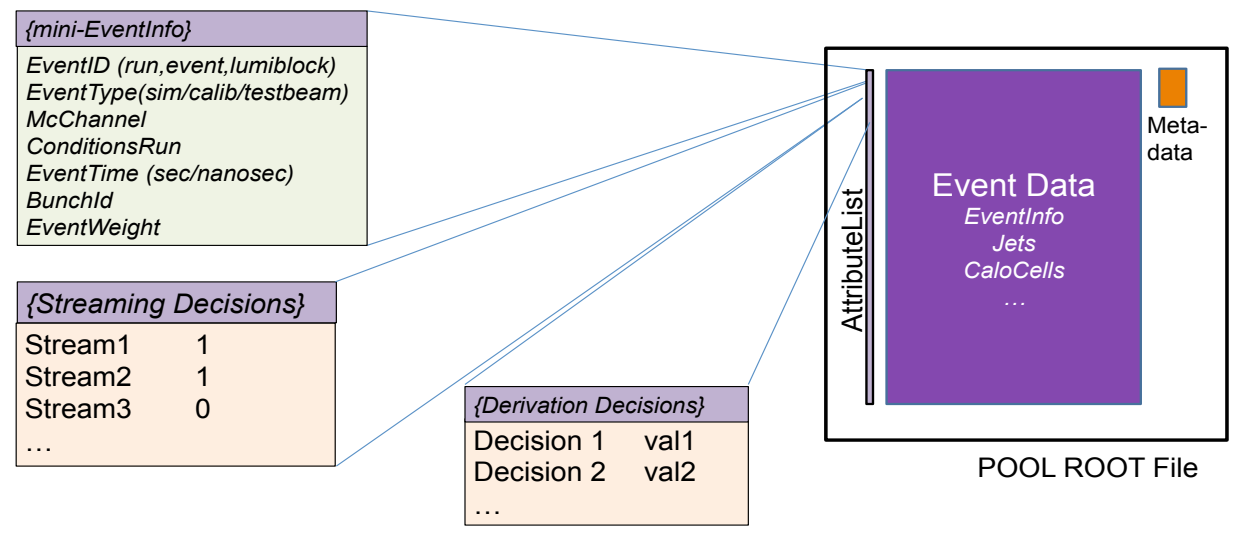

Figure 3. Out-of-band Event-level metadata in a data file for fast Event selection on input.

\subsection{Compression optimization for efficient selective reading}

The decision to combine all derivation streams together in the same file (described in the previous section) introduces a change in the reading pattern. Previously, reading from files containing already preselected events (skimmed files) was mostly sequential. Reading from a combined file containing both interesting and uninteresting events - for a particular analysis - becomes sparse reading, with an expected filtering of $0.1-5 \%$. Such selective reading of objects from ROOT files can be less efficient than sequential access, due to reading and decompressing entire buffers, which contains multiple events, even when only a fraction of that data had been requested by the application.

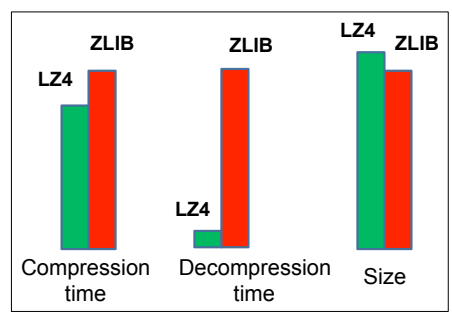

Figure 4. Relative performance comparison between LZ4 and ZLIB compression algorithms (in arbitrary units) for ATLAS Event data files

During Run 1 and Run 2, ATLAS was using the standard ROOT compression algorithm ZLIB for their derivation stream data. For Run 3, we evaluated another algorithm - LZ4 supported by the recent ROOT releases and the default in the new releases. We found that LZ4, thanks to it's very fast decompression (Figure 4), makes it possible to read data at $40 \%-100 \%$ of the rate of pre-selected samples (Figure 5), much faster than with ZLIB, while retaining most of the size reduction advantages of ZLIB compression. 


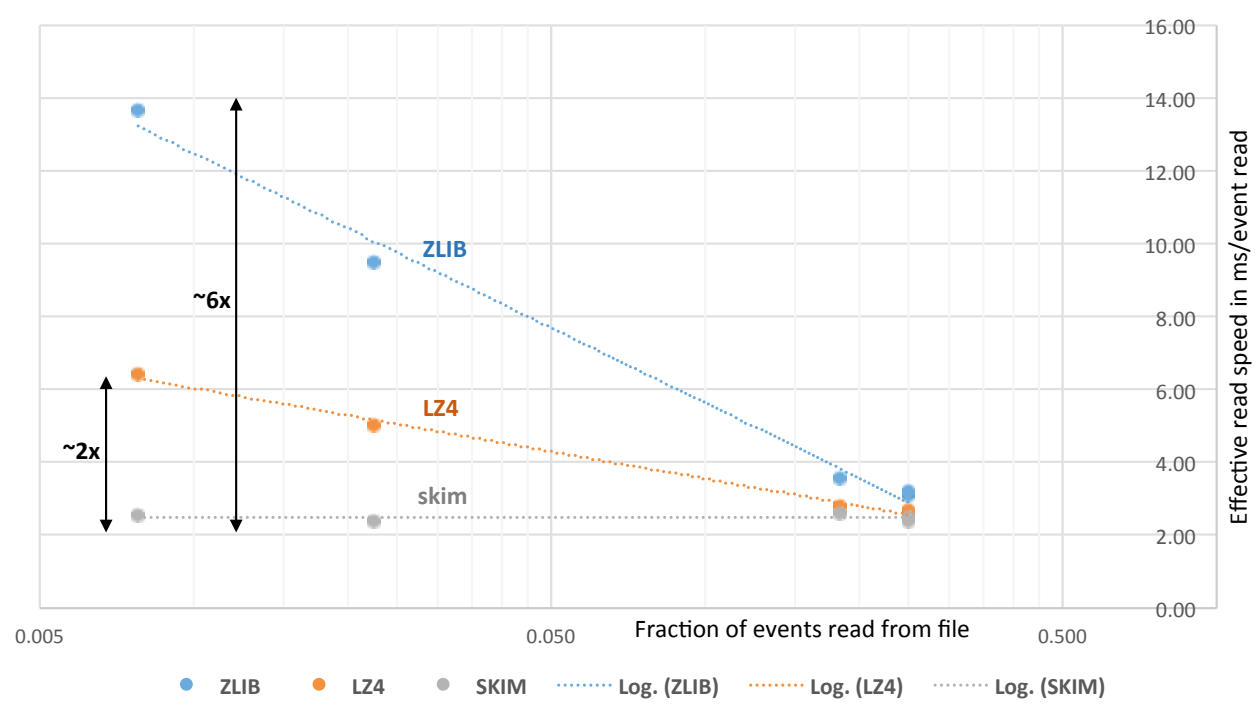

Figure 5. Effects of different compression algorithms on selective reading performance compared to $100 \%$ read (skim). The read time per event is constant for skimmed data file as it contains only preselected events. Read time per event goes up as selectivity increases for both ZLIB and LZ4 because there are less and less relevant events in every decompressed block.

\section{Conclusions}

ATLAS Computing is undergoing important changes to meet the challenges of LHC Run 3 data handling and processing. The offline software framework Athena, and in particular its I/O components, are evolving to support these changes. The framework foundations have gained capacity to work in a multi-threaded environment, which in turn provides the grounds for adaptation and testing of physics algorithms and other framework components. Object referencing was made more robust, in particular with respect to file merging. Data storage format and organization was modified with the intent to achieve better balance between disk space and performance. The work on the I/O layer continues to increase concurrency on writing and to gain performance by introducing more fine-grained locking in the Athena central $\mathrm{I} / \mathrm{O}$ services and concurrency for compression

\section{Acknowledgements}

The work is funded in part by the U.S. Department of Energy, Office of Science and High Energy Physics contracts.

\section{References}

1. ATLAS Collaboration (2008) "The ATLAS Experiment at the CERN Large Hadron Collider", J. Inst. 3, S08003 https://iopscience.iop.org/article/10.1088/1748-0221/3/08/S08003

2. ATLAS Collaboration (2019) Athena (Version 22.0.1) Zenodo http://doi.org/10.5281/zenodo.2641997 
3. G. A. Stewart et al (2016) "Multi-threaded software framework development for the ATLAS experiment" J. Phys.: Conf. Ser.762 012024

https://iopscience.iop.org/article/10.1088/1742-6596/762/1/012024

4. R. Brun, F. Rademakers (1996) "ROOT: An Object Oriented Data Analysis

Framework” AIHENP'96 Workshop Nucl. Inst. \& Meth. In Phys. Res. A 389 81-86.

http://root.cern.ch

5. D. Duellmann at al (2003) "The POOL data storage, cache and conversion mechanism" eConf C0303241 MOKT008

https://inspirehep.net/literature/620943

6. D. Riley, C. Jones (2019) “Multi-threaded Output in CMS using ROOT” EPJ Web

Conf., 21402016

https://www.epj-

conferences.org/articles/epjconf/abs/2019/19/epjconf_chep2018_02016/epjconf_chep2

018_02016.html

7. C. Bozzi et al (2017) "The LHCb software and computing upgrade forRun 3:

opportunities and challenges" J. Phys.: Conf. Ser.898 11200

https://iopscience.iop.org/article/10.1088/1742-6596/898/11/112002

8. J. Elmsheuser et al (2020) "Evolution of the ATLAS analysis model for Run-3 and prospects for HL-LHC” CHEP 2019 Proceedings 